\title{
Transcriptional response of human articular chondrocytes treated with fibronectin fragments: an in vitro model of the osteoarthritis phenotype
}

\author{
${ }^{1}$ Thurston Arthritis Research Center, University of North Carolina, Chapel Hill, North Carolina, USA \\ ${ }_{2}^{2}$ Curriculum in Genetics and Molecular Biology, University of North Carolina, Chapel Hill, NC 27599, USA \\ ${ }^{3}$ Division of Rheumatology, Allergy and Immunology, University of North Carolina, Chapel Hill North Carolina, USA \\ ${ }^{4}$ Department of Pediatrics, Rush University Medical Center, Chicago, Illinois, USA \\ ${ }^{5}$ Department of Cell Biology and Physiology, University of North Carolina, Chapel Hill North Carolina, USA \\ ${ }^{6}$ Curriculum in Bioinformatics and Computational Biology, University of North Carolina at Chapel Hill, Chapel Hill, NC 27599, USA; \\ ${ }^{7}$ Lineberger Comprehensive Cancer Center, University of North Carolina at Chapel Hill, Chapel Hill, NC 27599, USA.
}

Kathleen S. M. Reed ${ }^{1,2}$, Veronica Ulici ${ }^{1,3}$, Cheeho Kim ${ }^{1,3}$, Susan Chubinskaya ${ }^{4}$, Richard F. Loeser ${ }^{1,3, *}$, Douglas H. Phanstiel ${ }^{1,2,5,6,7, *}$

\section{Summary}

Objective: Fibronectin is a matrix protein that is fragmented during cartilage degradation in osteoarthritis (OA). Treatment of chondrocytes with fibronectin fragments (FN-f) has been used to model OA in vitro, but the system has not been fully characterized. This study sought to define the transcriptional response of chondrocytes to FN-f, and directly compare it to responses traditionally observed in OA.

Design: Normal human femoral chondrocytes isolated from tissue donors were treated with either FN-f or PBS (control) for 3, 6, or 18 hours. RNA-seq libraries were compared between time-matched FN-f and control samples in order to identify changes in gene expression over time. Differentially expressed genes were compared to a published OA gene set and used for pathway, transcription factor motif, and kinome analysis.

Results: FN-f treatment resulted in 1,224 differentially expressed genes over the time course. Genes that are up- or downregulated in OA were significantly up- $(\mathrm{p}<0.00001)$ or downregulated $(\mathrm{p}<0.0004)$ in response to FN-f. Early response genes were involved in proinflammatory pathways and their promoters were enriched for NF-kB-related motifs, whereas many late response genes were involved in ferroptosis, and their promoters were enriched for Jun-related motifs. Highly upregulated kinases included CAMK1G, IRAK2, and the uncharacterized kinase DYRK3, while growth factor receptors TGFBR2 and FGFR2 were downregulated.

Conclusions: FN-f treatment of normal human articular chondrocytes recapitulated many key aspects of the OA chondrocyte phenotype. This in vitro model is promising for future OA studies, especially considering its compatibility with genomics and genome-editing techniques.

\section{Introduction}

Osteoarthritis (OA) is the most common form of joint disease and affects over 250 million people worldwide, including over $10 \%$ of those older than 60 years ${ }^{1}$. There is no known cure, and treatments are currently limited to symptom management. One major reason for the lack of treatments is an incomplete understanding of the mechanisms that promote OA and its progression. While mouse models and human samples have provided valuable insights into OA biology, new human disease models amenable to manipulation and high-throughput screening would improve our ability to understand and potentially better treat this painful and disabling disease.

OA involves many, if not all, of the tissues that comprise articular joints, with the degradation and loss of articular cartilage noted as a central feature ${ }^{2}$. Studies of potential OA pathways often compare chondrocytes isolated from normal cartilage obtained from various animal species, including humans, to chondrocytes obtained from OA tissue. A limitation, particularly with human tissue, is that the OA chon- drocytes are most often isolated from cartilage obtained at the time of joint replacement, resulting in comparisons being made to cells at an advanced stage of disease. Animal models, including mice, have been critical for mechanistic studies, but major differences in genomes, body structures, and OA prevalence limit the relevance to human biology ${ }^{3}$.

A commonly used option for modeling the chondrocyte OA phenotype has been to stimulate primary cells or cell lines ex vivo with cytokines such as IL-1 or $\mathrm{TNFa}^{4}$. A major limitation of these studies is that the cells are treated with levels of cytokines in the $\mathrm{ng} / \mathrm{ml}$ range to obtain a desired response, while (at least in the synovial fluid) IL-1 and TNFa are only present in $\mathrm{pg} / \mathrm{ml}$ amounts ${ }^{5}$. In addition, recent studies, including failed clinical trials of IL-1 and TNFa inhibition in OA, suggest that multiple pro-inflammatory mediators contribute to OA development, and IL-1 or TNFa may not be the driving factors ${ }^{6-8}$.

An alternative in vitro model for simulating a chondrocyte OA phenotype utilizes fragments of fibronectin. Fibronectin is an extracellular matrix protein present in cartilage that is upregulated in OA tissue and subsequently

${ }^{*}$ Corresponding authors: Richard F. Loeser, MD, Thurston Arthritis Research Center, Division of Rheumatology, Allergy and Immunology, University of North Carolina School of Medicine, Campus Box 7280, Chapel Hill, North Carolina, 27599-7280, USA, richard_loeser@med.unc.edu

Douglas H. Phanstiel, PhD, Department of Cell Biology and the Thurston Arthritis Research Center, University of North Carolina School of Medicine, Campus Box 7280, Chapel Hill, North Carolina, 27599-7280, USA, douglas_phanstiel@med.unc.edu 
degraded by several proteases 9,10 . Fibronectin fragments (FN-f) of various sizes and at levels in the $\mu \mathrm{M}$ range have been detected in OA cartilage and synovial fluid as well as in cartilage from patients with rheumatoid arthritis ${ }^{11-13}$. Injection of FN-f into rabbit joints was found to induce cartilage proteoglycan loss, which is a feature of early $\mathrm{OA}^{14}$. Treatment of isolated human chondrocytes or cartilage explants with FN-f has been shown to recapitulate many known features of OA, including production of multiple matrix-degrading enzymes and proinflammatory cytokines found in OA joints ${ }^{9,15,16}$. While these results demonstrate the value of FN-f treatment for studying OA, the global similarity between FN-f-treated chondrocytes and OA chondrocytes has not been fully explored.

The purpose of this study was to characterize the transcriptional response to FN-f stimulation of ex vivo human chondrocytes and to compare this response to those previously observed in OA. We found that FN-f triggers a robust transcriptional response in primary chondrocytes, which correlates with changes observed during OA. Analysis of gene ontology terms, signaling pathways, and transcription factor motifs revealed that known regulators of OA progression also play a role in the FN-f response, as do a host of genes and pathways that had not previously been implicated in OA. These results support FN-f treatment as a viable model for studying transcriptional control of OA progression and provide a valuable resource for future studies.

\section{Methods}

\section{Sample collection and treatment}

Primary articular chondrocytes were isolated by enzymatic digestion from normal human femoral cartilage obtained from three tissue donors without a history of arthritis and with ages from 50-61 years, as previously described ${ }^{17}$. Cells were cultured to confluency in standard media with $10 \%$ fetal bovine serum and then made serum-free for 2 hours prior to treatment with a purified $42 \mathrm{kDa}$ endotoxin-free recombinant FN-f (1 $\mu \mathrm{M}$ in PBS), prepared as previously described, or PBS as a control ${ }^{18}$. The FN-f used here consists of domains 7-10 in native fibronectin which contains the RGD cell-binding domain recognized by the a $5 \beta 1$ integrin. After 3 , 6, or 18 hrs of treatment with FN-f or PBS, media was removed, cultures were quickly rinsed with cold PBS, and RNA was immediately isolated using the RNeasy kit from Qiagen.

\section{RNA-seq library preparation}

Prior to library preparation, all RNA samples were analyzed using a Tapestation RNA HS tape to confirm RNA integrity numbers (RIN) were within 8.5-10, which indicates high quality, intact RNA. Ribosomal RNA was removed using the New England Biolabs NEBNext rRNA Depletion Kit (Human/ Mouse/Rat), and RNA-seq libraries were prepared using the NEBNext Ultra II Directional RNA Library Prep Kit and NEBNext Multiplex Oligos for Illumina. Final libraries were then quantified using a Qubit 4 Fluorometer and run on a Tapestation D1000 HS tape, to confirm average fragment sizes were within $260-320$ base pairs and calculate molarity for pooling.

\section{Processing of RNA-Seq libraries}

RNA-seq libraries were sequenced to an average depth of approximately 58 million reads per sample (50 base pairs, paired-end reads) on an Illumina HiSeq 4000 (High Output). Low-quality reads and adapters were trimmed using Trim Galore! (v. 0.4.3), and trimmed reads were then quantified using Salmon quasi-mapping (v. 0.8.2). Both programs were run with default settings.

\section{Identifying differential genes}

Gene-level quantifications were summarized from each sample using tximport (v. 1.2.0). Differential analysis was conducted in $\mathrm{R}$ with DESeq2 (v. 1.22.2) using a design adjusting for donor variability when calculating differences between treatment groups ( donor + treatment). Differential genes were defined as genes with an FDR-adjusted p-value below .01 (Wald test) and an absolute fold-change above 2 when comparing FN-f treated samples to their timematched controls.

\section{Temporal clustering of genes}

To assign temporal response classes for the 1,224 differential genes, first a z-score was calculated from the variance-stabilized counts, centering the counts in each sample relative to the average counts among all samples for each gene. Then, for each donor sample, the untreated control score was subtracted from the FN-f treated score for every gene. The difference in z-score was then averaged over the three donors, ultimately providing three values for every gene representing the normalized expression relative to the control at each time point (3, 6, and 18 hours). This matrix was then clustered using k-means clustering with a $k$ of 4 . These clusters were labeled "Up Early", "Up Late", "Down Early", and "Down Late" based on their expression relative to untreated controls at each time point.

\section{Comparing with genes differentially expressed in OA cartilage}

The previously published RAAK study identified genes that were up- or downregulated in OA-affected cartilage compared to preserved cartilage in the same joint ${ }^{19}$. To focus on only the genes that exhibited the strongest changes in OA, the genes from the RAAK study were filtered to include only those that had a p-value of less than 0.01 and an absolute fold change of greater than 1.5. A Mann-Whitney U test was used to determine if the FN-f-induced fold change of each set of OA-responsive genes were significantly higher or lower, respectively, than the FN-f-induced fold change of genes outside of each gene set. 
bioRxiv preprint doi: https://doi.org/10.1101/2020.06.18.155390; this version posted June 18, 2020. The copyright holder for this preprint (which was not certified by peer review) is the author/funder, who has granted bioRxiv a license to display the preprint in perpetuity. It is made available under aCC-BY-NC-ND 4.0 International license.

\section{GO, KEGG \& Transcription Factor Motif Enrichment Analysis}

The "findMotifs.pl" tool in the HOMER software suite (v. 4.10.4) was used on each cluster of genes in order to identify enriched Gene Ontology (GO) terms, Kyoto Encyclopedia of Genes and Genomes (KEGG) pathways, and transcription factor motifs. Using the "-mknown" option, known transcription factor motifs were detected from the core mononucleotide human HOCOMOCO dataset (v. 11, p-value $<0.001$ ). Transcription factor families were then identified via TFClass ${ }^{20}$. De novo motifs with $\mathrm{p}$-values below $1 \times 10^{-8}$ and best-match motif scores below 0.6 were classified as "unannotated", in that they did not appear to have a conclusive known motif match.

\section{Kinome Visualization}

To identify and visualize protein kinases present in each cluster, the cluster assignments were plotted using the human kinome visualization tool, Coral ${ }^{21}$. Flat text files were created listing the ENSEMBL ID, k-means cluster, and maximum absolute fold change among all three time points for each differential gene. These lists were then used to plot both categorical (cluster, encoded in branch/node color) and qualitative data (maximum absolute fold change, encoded in node size) on the protein kinase tree, originally published by Manning et $\mathrm{al}^{22}$.

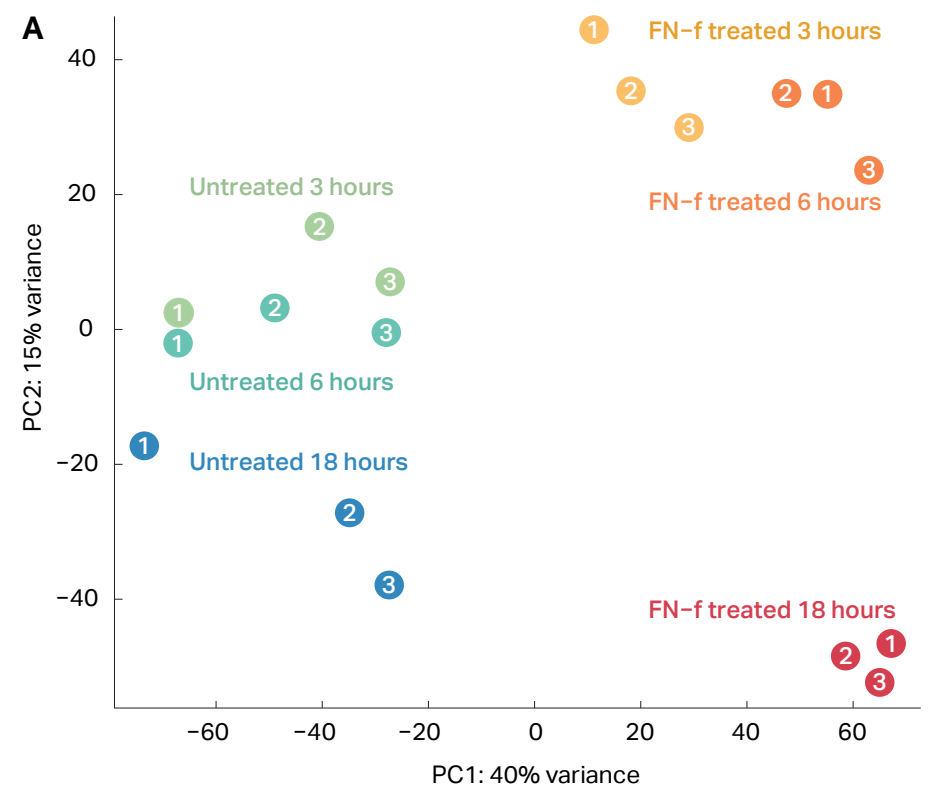

\section{Data Availability}

Raw sequencing data, transcript-level quantification output from Salmon, and a table containing gene-level summaries of read counts in each sample, as well as Wald test p-values, log2 fold change, and normalized count z-score at each time point, are made publicly available at GEO accession GSE150411.

\section{Results}

$F N-f$ induces global changes in chondrocyte gene expression

To determine the extent to which FN-f treatment alters transcription in human chondrocytes, we performed a threepoint RNA-seq time course of 3, 6, and 18 hours (Fig S1). We used principal component analysis (PCA) of the 18 samples to determine the extent to which each attribute (donor, time in culture, and time treated with FN-f) contributed to transcriptional state. Untreated samples clustered largely by donor rather than by time in culture, suggesting that time in culture had only minor impacts on gene expression (Fig 1A). Conversely, samples treated with FN-f clustered by treatment time, suggesting that FN-f treatment played a larger role in transcriptional state than the genotype of the donor cells. Statistical analysis of differential expression patterns confirmed these results. Untreated samples

\section{B Number of genes that increase or decrease in response to}

\section{Time in culture}

or

\section{FN-f treatment}

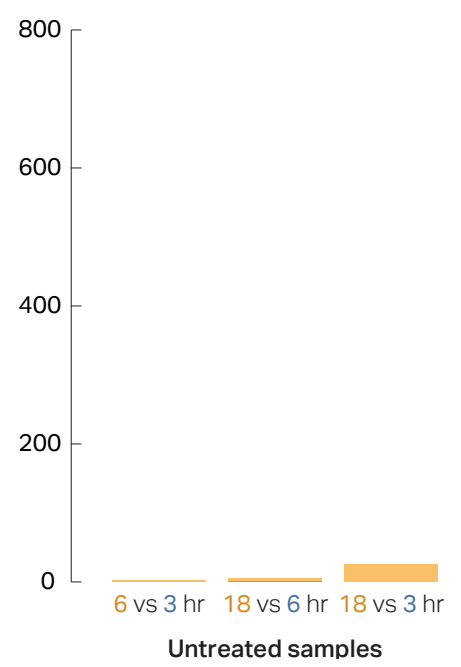

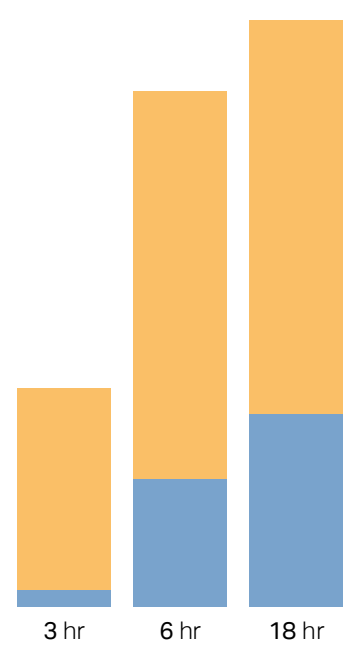

FN-f vs untreated samples

Figure 1. FN-f treatment induces a robust transcriptional response in human chondrocytes. (A) Principal component analysis (PCA) of each sample (colors indicate condition, numbers indicate donor) reveals a distinct separation between FN-f-treated and untreated samples along the first principle component (explaining $40 \%$ variance). Additionally, FN-f-treated samples cluster by length of treatment rather than by donor, whereas untreated samples cluster more based on donor (particularly along PC1). (B) Bar plots depicting the number of genes that exhibit significant differences in expression due to time in culture (left) or FN-f treatment (right; Wald test FDR $>0.01$, absolute fold change $>2$ ). Yellow and blue bars represent the number of genes that increase or decrease in each comparison. (Left) A bar plot depicting the number of genes that change significantly between untreated control samples reveals that time in culture has only a minimal impact on gene expression. (Right) A bar plot depicting the number of genes that change significantly in FN-f-treated samples compared to time-matched controls demonstrates that FN-f induces a robust transcriptional response in chondrocytes. 
bioRxiv preprint doi: https://doi.org/10.1101/2020.06.18.155390; this version posted June 18, 2020. The copyright holder for this preprint (which was not certified by peer review) is the author/funder, who has granted bioRxiv a license to display the preprint in perpetuity. It is made available under aCC-BY-NC-ND 4.0 International license.

exhibited only 28 differentially expressed genes between time points (FDR $<0.01$, fold change $>2$; Fig 1B). In contrast, comparison of FN-f-treated samples to their time-matched controls revealed 1,224 genes that changed significantly in response to FN-f treatment in at least one of the three time points (Table S1). Increased treatment time correlated with increased numbers of differentially expressed genes, with 317,747 , and 850 genes affected at 3, 6, and 18 hours, respectively. Together, these results demonstrate that FN-f treatment has a profound effect on transcription that is distinct from the effects of ex vivo culturing, and this effect is robust when accounting for variation in response among biological replicates.

Investigation of specific differential genes revealed expected changes for many known regulators of OA. Genes upregulated in response to $\mathrm{FN}-\mathrm{f}$ included cytokines and chemokines such as CXCL2 (189-fold), LIF (160-fold), and IL6 (87-fold). Interleukin-1ß (IL1B), a proinflammatory cytokine with elevated expression in OA chondrocytes ${ }^{23,24}$, was upregulated at all time points, peaking at over 100-fold. Upregulated genes also included proteases such as MMP13 and MMP10 (both 12-fold). Matrix metallopeptidase 13 (MMP13) is an enzyme that degrades type II collagen and is thought to play a critical role in cartilage degradation in $\mathrm{OA}^{12,15,25-28}$. Interestingly, among the downregulated genes were the collagen-binding integrins ITGA10 and ITGA11, which decreased 3.7- and 3.5-fold, respectively.

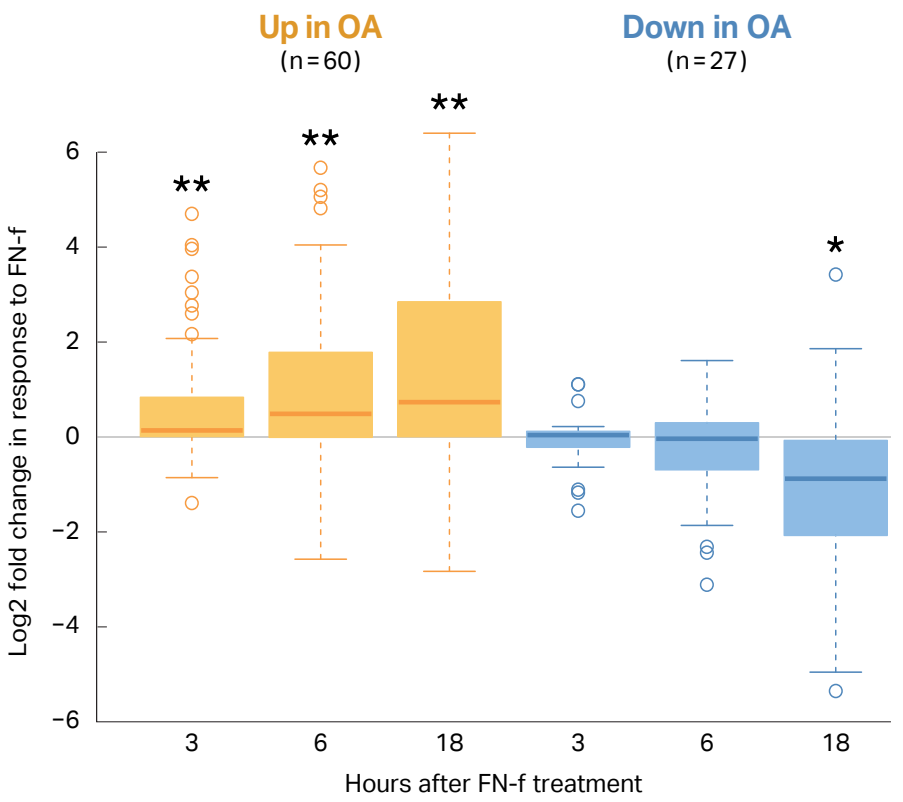

Figure 2. FN-f treatment induces changes similar to osteoarthritis. The previously published RAAK study compared osteoarthritis cartilage to preserved cartilage in the same joint, and of the differential genes reported, 60 were upregulated and 27 were downregulated with a high degree of change and significance (FDR p-value $<0.01$, absolute fold-change $>1.5$ ). The boxplot shows the log2 fold-change between FN-f treated and control samples at each time point for both the up(yellow) and downregulated (blue) genes. These genes were ranked by fold-change and compared to a ranked list of fold-changes for genes outside of the OA set (Mann-Whitney p-values; ${ }^{* *}=<0.00001,{ }^{*}=0.0004$ )
FN-f treatment induces transcriptional changes similar to $O A$

Changes in gene expression in response to FN-f were compared to the differential expression reported in chondrocytes isolated from OA and preserved tissue in the RAAK study ${ }^{19}$ using a subset of differential genes with the strongest effects (p-value < 0.01, absolute fold change > 1.5) (Table S2; Fig 2). Genes upregulated in the OA tissue were also upregulated in response to FN-f treatment (Mann-Whitney U test, p-value $<0.00001$ ), and these effects grew more pronounced with longer exposure to FN-f (Fig 2). Genes that were downregulated in OA tissue were similarly downregulated in response to FN-f, though only exhibiting statistical significance after 18 hours of treatment (Mann-Whitney U test, p-value = 0.0004). These results suggest that FN-f induces similar transcriptional changes to those found in OA chondrocytes.

To further evaluate FN-f treatment as a model of the OA chondrocyte phenotype, we compared our differentially expressed genes to genes that have been implicated in OA by Genome Wide Association Studies (GWAS). Tachmazidou et al. ${ }^{29}$ recently identified 64 loci harboring OA-associated variants. These single-nucleotide polymorphisms (SNPs) represent regions with genotypes statistically associated with the OA phenotype, but most occur in non-coding regions of the genome rather than in gene bodies. This suggests that the SNPs likely impact regulatory regions, and the gene(s) they affect-which in turn promote the OA phenotype-could be hundreds of thousands of base pairs away. In order to identify genes that might be affected by these OA variants, we overlapped the locations of the SNPs with our differentially expressed genes and found that 72 of the genes identified here were within $500 \mathrm{~Kb}$ of a GWAS SNP. These genes are summarized in Table 1 and include many genes with previously reported connections to OA, such as $N F K B 1^{30}$, SOX $7^{31}, I L 11^{32}, G D F 5^{33,34}$, FGF18 ${ }^{35}$, TNFSF15, and NKX3-2 ${ }^{36}$.

\begin{tabular}{llll|l}
\multicolumn{2}{c}{ Up Early } & Up Late & Down Early & Down Late \\
ALCAM & PSMB9 & AHCYL2 & ARVCF & ANGPTL2 \\
BMP5 & RELA & AQP9 & CATSPER1 & C1QL1 \\
C22orf23 & RTP4 & BTN2A1 & CLDN23 & CISH \\
CLIC5 & SCARF1 & BTN2A2 & CLEC18A & COL21A1 \\
DAXX & SLC30A10 & BTN3A2 & FAM53A & CPA4 \\
FGF18 & SOX7 & BTN3A3 & GDF5 & EPB41L1 \\
FKSG63 & TAP1 & GINS3 & KANK2 & ERG \\
FOSL1 & TNFSF15 & HIC1 & LRIG3 & HIST1H2AB \\
HAS3 & & IL11 & NKX3-2 & LBH \\
ICAM1 & & ITGB3 & PLCD3 & MEST \\
ICAM4 & & NQO1 & PPP1R3B & NIPSNAP3B \\
ICAM5 & & PAPPA & SEPT5 & PDK4 \\
IER2 & & PDXP & SERTAD4 & RTN4RL1 \\
LDLR & & RILP & SMAD6 & SGK223 \\
MICALL1 & & SLC39A8 & WWP2 & SLC29A1 \\
NFKB1 & & SLC43A2 & & \\
NFKBIE & & TAP2 & &
\end{tabular}

Table 1. FN-f responsive genes near osteoarthritis GWAS loci. This table lists all differential genes that change in response to FN-ftreatment and that have a transcriptional start site within $500 \mathrm{~Kb}$ of a GWAS SNP as identified in Tachmazidou et al, 201929. For each gene, the response class is listed (as determined by k-means clustering of all differential genes). 
bioRxiv preprint doi: https://doi.org/10.1101/2020.06.18.155390; this version posted June 18, 2020. The copyright holder for this preprint (which was not certified by peer review) is the author/funder, who has granted bioRxiv a license to display the preprint in perpetuity. It is made available under aCC-BY-NC-ND 4.0 International license.

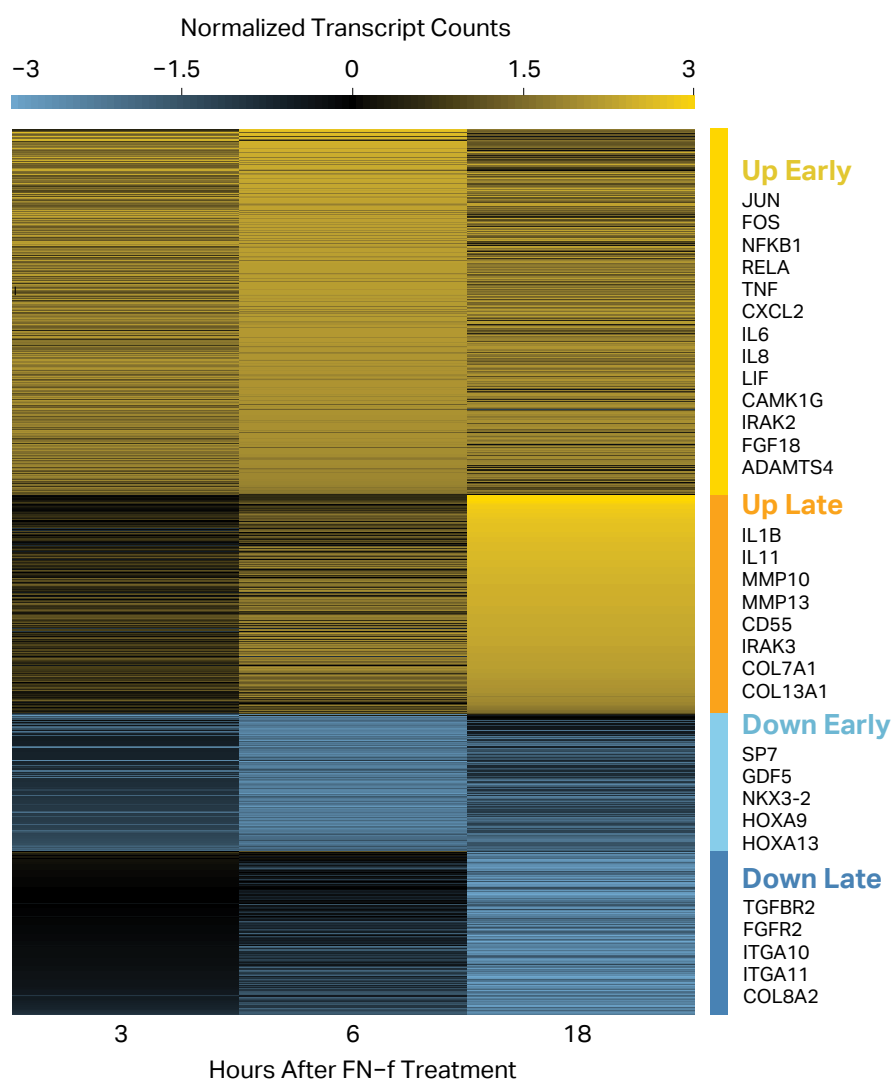

Figure 3. FN-f treatment regulates both early- and late-response genes. The genes that changed significantly in response to fibronectin fragment treatment were clustered according to their difference in z-score normalized counts between FN-f-treated and untreated samples at each time point. This separated the differential genes into four classes: "Up Early" (yellow; $n=506$ ), "Up Late" (orange; $n=302$ ), "Down Early" (light blue; n=190), and "Down Late" (dark blue; n=226). Selected genes are highlighted in each cluster.
These results reinforce the validity of this model for studying OA-related processes and could help identify the genes affected by OA-associated genetic variants.

\section{FN-f triggers both early- and late-response genes}

To investigate the temporal patterns of transcriptional changes in response to FN-f, we performed k-means clustering of differentially expressed genes (Fig 3). This revealed four distinct temporal patterns. Early-response genes (both up- and downregulated) exhibited changes in expression as early as 3 hours but generally peaked at 6 hours of FN-f treatment. These early-response genes include many upregulated genes that have been previously implicated in OA, including AP-1 components FOS and JUN ${ }^{24,37}, N O D 2^{38}$, RIPK2 ${ }^{38}$, the aggrecanase ADAMTS4 ${ }^{24,39}, C X C L 1, C X C L 2$, and CXCL ${ }^{16}$, LIF, TNF ${ }^{40-42}$, TNFAIP6 and TNFRSF $11 B^{19}$, PTGES $^{19}$, $I L 6$ and $I L 8^{39,41,43,44}$. Among genes that decreased early was the transcription factor SP7, which is downregulated by $\mathrm{TNF}^{45}$. Late-response genes showed maximum absolute fold changes after 18 hours of FN-f treatment. Many of these genes have also been implicated in OA and/or matrix remodeling, including $M M P 1, M M P 10$, and $M M P 13^{15,38,46}, C D 55$, $N G F$ and $P A P P A^{19}$, several interleukins such as IL1B, IL11 and $I L 17 C^{39,43,47}$, the interleukin-receptor-associated kinase IRAK3, the IL1 receptor antagonist IL1RN, and collagens CO$L 13 A 1$ and COL7A1 ${ }^{48,49}$. The cartilage-specific integrin a10 1 (ITGA10) as well as integrin a11ß1 (ITGA11) were both downregulated at late time points ${ }^{50}$. These results highlight the value of looking at FN-f response across a time course as it both reveals transient events not observed at every time point and provides insight into the temporal order and possibly even causal relationships between regulatory events.
A

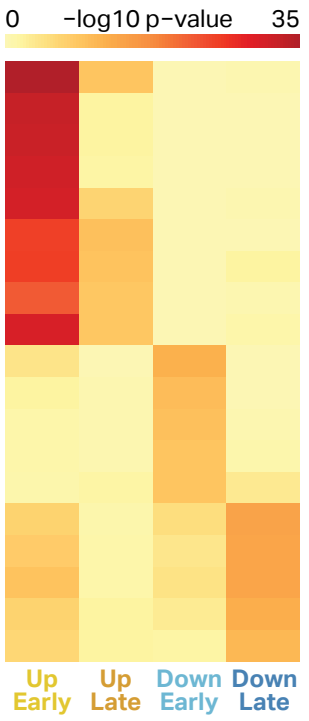

\section{GO Terms}

response to cytokine

response to other organism

response to external biotic stimulus

response to biotic stimulus

inflammatory response

immune system process

cellular response to chemical stimulus response to chemical

response to organic substance

regulation of transcription by RNA polymerase II embryo development

embryonic organ development

embryonic morphogenesis

skeletal system development

system development

anatomical structure development

multicellular organism development

developmental process

multicellular organismal process
B

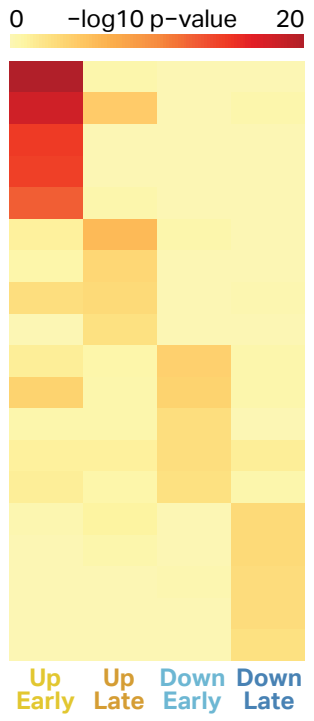

\section{KEGG Pathways}

TNF signaling pathway

cytokine-cytokine receptor interaction

influenza A

NF-kappa B signaling pathway

measles

ferroptosis

beta-Oxidation, acyl-CoA synthesis

fluid shear stress and atherosclerosis protein digestion and absorption

TGF-beta signaling pathway transcriptional misregulation in cancer

signaling pathways regulating pluripotency of stem cells proteoglycans in cancer

Hippo signaling pathway

ECM-receptor interaction

focal adhesion

dilated cardiomyopathy

regulation of actin cytoskeleton

arrhythmogenic right ventricular cardiomyopathy (ARVC)

Figure 4. Gene ontology terms and KEGG pathways enriched in response to FN-f. For every cluster of differential genes, the top five GO terms (A) and KEGG pathways (B) were selected based on enrichment p-value. Heatmap color represents the -log10 of the enrichment p-value for each term or pathway for genes in each cluster (up early, up late, down early, and down late). 
We investigated whether the length of genes-and the corresponding time it would take to transcribe them-could account for the difference in response time. While the mean gene length for the late-response genes was statistically significantly longer than that of early-response genes (Mann-Whitney U test, p-value = 4e-6; Fig S2), the two distributions exhibited substantial overlap. Therefore, gene length is not likely to be the primary determinant of early vs late response.

Fn-f induces transcription of proinflammatory genes and pathways

To understand the likely phenotypic impact of the changes induced by FN-f, we performed Gene Ontology (GO) enrichment analysis for the genes in each of the four clusters (Fig 4A). Both early- and late-response genes that were upregulated in response to FN-f were strongly enriched for proinflammatory biological processes including "response to cytokine", "inflammatory response", and "immune system process". Genes in these categories include NF-kB subunits, chemokine receptors, interleukins, MAP kinases, TNF ligands, and other cytokines. This is consistent with previous studies that have demonstrated that FN-f treatment stimulates a proinflammatory response via MAP kinases and NF- $\kappa B$ signaling ${ }^{15,16,25,41,51}$, as well as the established role of inflammation in the progression of $\mathrm{OA}^{24,30,39,40,42,43,47,52,53}$. Genes downregulated in response to FN-f were enriched for GO terms for development and morphogenesis and included HOX genes, TGFBR2, and COL8A2, among others.

To determine pathways that were affected by FN-f treatment, we identified Kyoto Encyclopedia of Genes and Genomes (KEGG) pathways that were enriched in each of the four gene sets (Fig 4B). Early-response upregulated genes were enriched for TNF and NF-kB pathways, both of which have been implicated in OA and even targeted for therapeutic OA treatments ${ }^{30,42,54}$. Intriguingly, late-response upregulated genes were most strongly enriched for the ferroptosis pathway, a form of programmed cell death dependent on iron and accumulation of lipid peroxides induced by reactive oxygen species, which have been implicated in $\mathrm{OA}^{55}$. The late-response upregulated genes involved in ferroptosis included ACSL1, GCLM, SLC39A8, HMOX1, ACSL5, FTH1, SLC7A11, and ACSL4. The downregulated genes were enriched for the TGF $\beta$ and Hippo signaling pathway as well as ECM-receptor and focal adhesion genes.

To visualize how specific kinases were regulated in response to FN-f, we generated kinome tree maps using the human kinase visualization tool Coral (Fig 5). Kinase branches and nodes were colored to indicate time course clusters. This analysis identified many kinases with suspected roles in OA progression, including the p38 pathway member MAP2K3, which was upregulated 9.6-fold in response to $\mathrm{FN}-\mathrm{f}^{41,56}$. In contrast, we found that TGFBR2 was downregulated 3.5-fold in response to FN-f, which is consistent with recent findings in which decreased TGFBR2 was correlated with increased OA severity in mice ${ }^{57}$. These studies also uncovered a num- ber of kinases that have not been previously implicated in OA or chondrocyte dysfunction. For example, DYRK3, which was upregulated 3.7-fold in response to FN-f, is relatively poorly annotated and has thus been characterized as part of the "dark kinome". DYRK3 and other understudied kinases identified in this study provide novel targets for further study with regard to their involvement in OA.

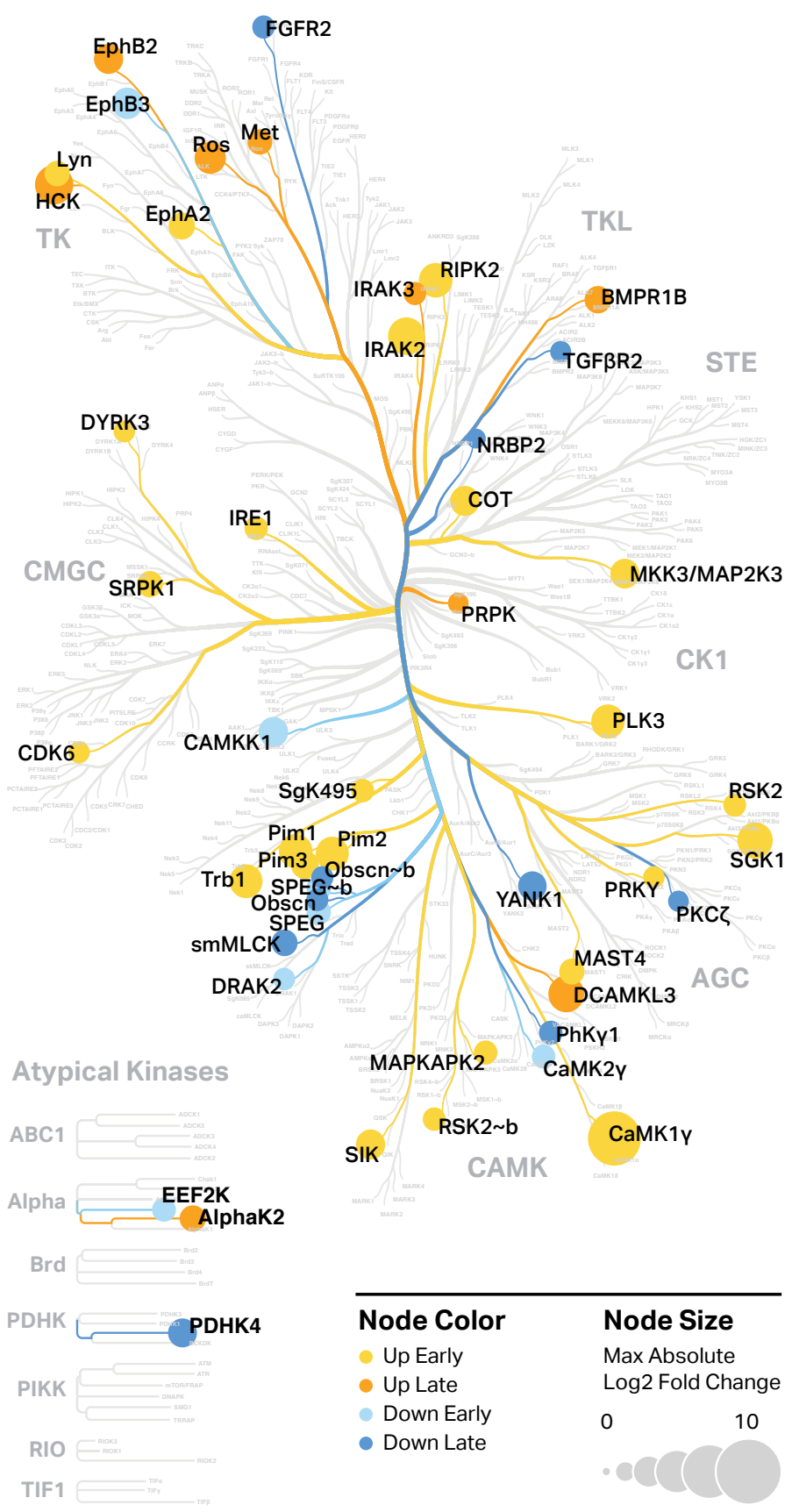

Figure 5. Protein kinases transcriptionally regulated by FN-f.

Protein kinases that are differentially expressed in response to FN-f treatment were highlighted in a human kinome map generated by Coral. Color represents the temporal response class of the genes as determined by k-means clustering, while node size represents the maximum log2 fold change between time-matched FN-f-treated and control samples among the three time points. 
bioRxiv preprint doi: https://doi.org/10.1101/2020.06.18.155390; this version posted June 18, 2020. The copyright holder for this preprint (which was not certified by peer review) is the author/funder, who has granted bioRxiv a license to display the preprint in perpetuity. It is made available under aCC-BY-NC-ND 4.0 International license.

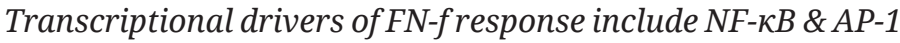

To determine the transcription factors that were responsible for the global transcriptional changes induced by FN-f treatment, we used the HOMER software suite to identify transcription factor motifs that were enriched in the promoters of genes for each of the four clusters (Fig 6). Upregulated early-response genes exhibited a strong enrichment for motifs associated with multiple members of the NF- $k B$ complex, including REL, RELB, TF65, and NFKB2. In contrast, upregulated late-response gene promoters were enriched for motifs of Jun-related proteins, including JUNB, NF2L2, and NFE2. JUNB is a member of the Activator Protein 1 (AP-1) which has been previously shown to contribute to $\mathrm{OA}$ and cartilage matrix degradation ${ }^{24}$. These findings are consistent with the fact that AP-1 and NF- $\kappa \mathrm{B}$ subunits are also upregulated in response to FN-f.

This analysis also revealed the enrichment of several unannotated motifs in the promoters of upregulated genes (Fig S3). The presence of these de novo motifs may suggest that transcription factors with currently uncharacterized motifs also play a role in FN-f response. Further studies investigating the proteomic and phosphoproteomic landscape of chondrocytes responding to FN-f treatment may help in the further characterization of this model system and identify other key regulators in this response, which could also play a role in OA progression.

\section{Discussion}

Developing cell culture systems that model key aspects of diseases can be incredibly valuable for deciphering mechanisms and testing therapeutic interventions, particularly when high-throughput screens are necessary. Using a fragment of the matrix protein fibronectin, we investigated a human cell-culture model of chondrocyte response to cartilage matrix breakdown, a key trigger of OA. Our transcriptome-wide analysis confirmed a similarity between this system and changes observed in OA tissue, suggesting that this is a powerful system with which to study the OA chondrocyte phenotype. We confirmed that many OA-responsive genes also change significantly in normal chondrocytes in response to FN-f. In addition, we classified over a thousand FN-f-responsive genes by their direction and timing of regulation and confirmed that many genes and pathways upregulated in response to FN-f have previously been characterized as a part of the OA phenotype. This includes inflammatory cytokines and chemokines such as $I L 1 B$ and CXC ligands, matrix-degrading proteinases such as MMP13, and members of the NF- $\kappa B$ signaling pathway.

The presence of early- and late-response gene clusters in this system is reminiscent of primary and secondary responses observed in other systems, such as the inflammatory response in immune cells $s^{58-63}$ and the growth factor response promoting differentiation and proliferation ${ }^{64}$. Variation in response times can stem from differences in genomic and regulatory features, including the degree to which products are regulated at transcriptional, post-transcriptional and translational levels, the baseline differences in RNA polymerase II occupancy at transcription start sites, and the dependency of target gene regulation on epigenetic modifications ${ }^{64}$. Further exploration of these and other regulatory mechanisms in this system may provide a deeper understanding of how chondrocytes are phenotypically altered in OA.

This study also revealed many genes and transcription factors that have not been previously associated with OA, suggesting new lines of interest for further investigation. One example is the finding of over-representation of the ferroptosis pathway in the upregulated late-response genes. Ferroptosis is a relatively recently described mechanism of cell death that involves iron and excessive levels of lipid peroxides generated by oxidation of lipids ${ }^{55}$. Ferroptosis can result from disturbances in the glutathione-dependent antioxidant system, release of excessive reactive oxygen species (ROS) from the mitochondria, and oxidation of lipids by lipoxygenases and cyclooxygenases ${ }^{65}$. Previous studies have demonstrated that FN-f treatment of chondrocytes generates ROS that regulate signaling involved in MMP expres$\operatorname{sion}^{18}$. Although ferroptosis per se has not been described in OA cartilage, studies have demonstrated lipid peroxida-

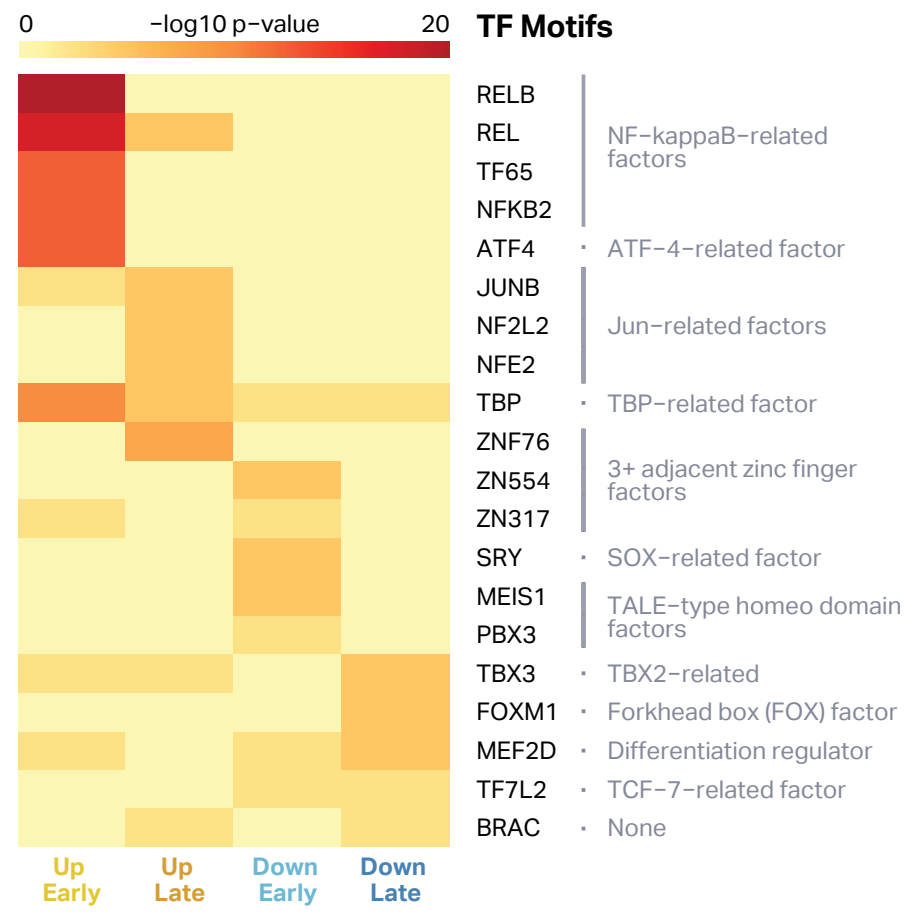

Figure 6. Transcription factor motifs enriched at FN-f early- and late-response genes. The promoters of genes in each cluster were analyzed for enriched transcription factor motifs from the HOCOMOCO database, and the top five from each cluster (based on enrichment p-value) are plotted in the heatmap. Transcription factor families from TFClass are listed in grey on the right ${ }^{20}$. Each temporal class of genes has unique transcription factor motifs enriched. The promoters of genes upregulated early are enriched for the motifs bound by various NFkB members, while the promoters of genes upregulated late are enriched for factors related to the AP-1 component Jun. 
tion $^{66}$, glutathione oxidation ${ }^{67}$, mitochondrial dysfunction ${ }^{68}$, and increased activity of lipoxygenases and cyclooxygenas$\mathrm{es}^{69}$, indicating ferroptosis could contribute to chondrocyte death in OA cartilage. This finding may also be relevant to osteoarthritis associated with hemochromatosis where excessive iron is present ${ }^{70}$.

The intersection between FN-f-responsive genes and OA GWAS loci provides a subset of genes that could be affected by OA-associated genomic variants, offering potential targets for follow-up studies. In addition to the NF- $\kappa$ B family, which has been considered as an OA target for quite some time, FGF18 was a FN-f-responsive gene (up early) present in the GWAS dataset. Unlike NF- $\kappa B, F G F 18$, which is an anabolic growth factor, is in clinical trials for knee $\mathrm{OA}$ as an intra-articular agent that may promote cartilage growth ${ }^{71}$. Additional growth factors present in both datasets were the BMP family members GDF5 (down early) and BMP5 (up early) as well as the BMP signaling protein SMAD6 (down early). Consistent with the FN-f-induced chondrocyte phenotype, allelic variation in the GDF5 gene has been associated with reduced expression ${ }^{72}$. BMP5 is a regulator of bone and cartilage formation during development, but its role in OA is not clear ${ }^{73}$. SMAD6 is an inhibitor of $S M A D 1 / 5$, and its overexpression in mice was associated with a reduction in osteophyte formation, suggesting that decreased SMAD6 expression could be detrimental in $\mathrm{OA}^{74,75}$.

While FN-f treatment of ex vivo chondrocytes represents a powerful tool to understand some of the events that promote OA, it does not recapitulate all aspects of OA, nor does it serve to replace animal models nor analysis of human tissue. Osteoarthritis is a complex disease involving multiple tissues and arises due to both genetic and environmental factors. A more complete mechanistic understanding of $\mathrm{OA}$ therefore requires orthogonal approaches with offsetting advantages and limitations. This ex vivo $\mathrm{FN}-\mathrm{f}$ treatment model does, however, fill a valuable gap and provide a flexible and manipulatable system with which to understand the behavior of chondrocytes in both healthy and disease conditions. By combining this system with recent advances in genomics and genome editing (including the ability to edit primary human chondrocytes ${ }^{76}$ ), this FN-f model offers incredible promise for study of OA.

\section{Acknowledgements}

We would like to thank the Gift of Hope Organ and Tissue Donor Network, and the donor families, for providing normal donor tissue. We would like to thank Dr. Arkady Margulis for donor tissue procurement and Mrs. Arnavaz Hakimiyan for technical assistance. We would also like to thank Erika Deoudes for figure and preprint design.

\section{Author Contributions}

K.S.M.R. and C.K. carried out the analysis and interpretation of the data.

K.S.M.R. and V.U. collected and assembled the data.

K.S.M.R., D.H.P., and R.F.L. drafted the manuscript.

C.K. and V.U. revised the article for important intellectual content.

R.F.L. and D.H.P. obtained funding for, conceived, and designed the experiments.

All authors provided final approval of the article prior to submission.

\section{Role of the Funding Source}

This project was supported by grants from the National Institute of Arthritis, Musculoskeletal, and Skin Disease (R37-AR049003), the National Institute on Aging (RO1AG044034), the National Human Genome Research Institute (R00-HG008662) and the National Institute of General Medical Sciences (R35-GM128645 and T32-GM007092). This project was also supported in part by the Klaus Kuettner Chair for Osteoarthritis Research (SC).

\section{Conflict of Interest}

The authors certify that they do not have any affiliations with or involvement in any organization or entity with financial or non-financial interest in the subject matter and materials discussed in this manuscript.

\section{References}

1. Hunter DJ, Bierma-Zeinstra S. Osteoarthritis. Lancet. 2019;393(10182):1745-1759.

2. Loeser RF, Goldring SR, Scanzello CR, Goldring MB. Osteoarthritis: a disease of the joint as an organ. Arthritis Rheum. 2012;64(6):1697-1707.

3. Bapat S, Hubbard D, Munjal A, Hunter M, Fulzele S. Pros and cons of mouse models for studying osteoarthritis. Clin Transl Med. 2018;7(1):36.

4. Johnson CI, Argyle DJ, Clements DN. In vitro models for the study of osteoarthritis. Vet J. 2016;209:40-49.

5. Sohn DH, Sokolove J, Sharpe O, et al. Plasma proteins present in osteoarthritic synovial fluid can stimulate cytokine production via Toll-like receptor 4. Arthritis Res Ther. 2012;14(1):R7.

6. Vincent TL. IL-1 in osteoarthritis: time for a critical review of the literature. F1000. 2019;8:934.

7. Fleischmann RM, Bliddal H, Blanco FJ, et al. A Phase II Trial of Lutikizumab, an Anti-Interleukin- $1 \alpha / \beta$ Dual Variable Domain Immunoglobulin, in Knee Osteoarthritis Patients With Synovitis. Arthritis Rheumatol. 2019;71(7):1056-1069. 
8. Kloppenburg M, Ramonda R, Bobacz K, et al. Etanercept in patients with inflammatory hand osteoarthritis (EHOA): a multicentre, randomised, double-blind, placebo-controlled trial. Ann Rheum Dis. 2018;77(12):17571764.

9. Homandberg GA. Potential regulation of cartilage metabolism in osteoarthritis by fibronectin fragments. Front Biosci. 1999;4:D713-D730.

10. Zack MD, Arner EC, Anglin CP, Alston JT, Malfait A-M, Tortorella MD. Identification of fibronectin neoepitopes present in human osteoarthritic cartilage. Arthritis Rheum. 2006;54(9):2912-2922.

11. Carnemolla B, Cutolo M, Castellani P, Balza E, Raffanti $\mathrm{S}$, Zardi L. Characterization of synovial fluid fibronectin from patients with rheumatic inflammatory diseases and healthy subjects. Arthritis Rheum. 1984;27(8):913921.

12. Xie DL, Meyers R, Homandberg GA. Fibronectin fragments in osteoarthritic synovial fluid. $J$ Rheumatol. 1992;19(9):1448-1452.

13. Homandberg GA, Wen C, Hui F. Cartilage damaging activities of fibronectin fragments derived from cartilage and synovial fluid. Osteoarthritis Cartilage. 1998;6(4):231-244.

14. Homandberg GA, Meyers R, Williams JM. Intraarticular injection of fibronectin fragments causes severe depletion of cartilage proteoglycans in vivo. J Rheumatol. 1993;20(8):1378-1382.

15. Forsyth CB, Pulai J, Loeser RF. Fibronectin fragments and blocking antibodies to alpha2beta1 and alpha5beta1 integrins stimulate mitogen-activated protein kinase signaling and increase collagenase 3 (matrix metalloproteinase 13) production by human articular chondrocytes. Arthritis Rheum. 2002;46(9):2368-2376.

16. Pulai JI, Chen H, Im H-J, et al. NF-kappa B mediates the stimulation of cytokine and chemokine expression by human articular chondrocytes in response to fibronectin fragments. J Immunol. 2005;174(9):5781-5788.

17. Loeser RF, Pacione CA, Chubinskaya S. The combination of insulin-like growth factor 1 and osteogenic protein 1 promotes increased survival of and matrix synthesis by normal and osteoarthritic human articular chondrocytes. Arthritis Rheum. 2003;48(8):2188-2196.

18. Wood ST, Long DL, Reisz JA, et al. Cysteine-Mediated Redox Regulation of Cell Signaling in Chondrocytes Stimulated With Fibronectin Fragments. Arthritis Rheumatol. 2016;68(1):117-126.

19. Ramos YFM, den Hollander W, Bovée JVMG, et al. Genes involved in the osteoarthritis process identified through genome wide expression analysis in articular cartilage; the RAAK study. PLoS One. 2014;9(7):e103056.
20. Wingender E, Schoeps T, Haubrock M, Krull M, Dönitz J. TFClass: expanding the classification of human transcription factors to their mammalian orthologs. Nucleic Acids Res. 2018;46(D1):D343-D347.

21. Metz KS, Deoudes EM, Berginski ME, et al. Coral: Clear and Customizable Visualization of Human Kinome Data. Cell Syst. 2018;7(3):347-350.e1.

22. Manning G, Whyte DB, Martinez R, Hunter T, Sudarsanam $S$. The protein kinase complement of the human genome. Science. 2002;298(5600):1912-1934.

23. Daheshia M, Yao JQ. The interleukin 1beta pathway in the pathogenesis of osteoarthritis. J Rheumatol. 2008;35(12):2306-2312.

24. Ji Q, Xu X, Zhang Q, et al. The IL-1ß/AP-1/miR-30a/ ADAMTS-5 axis regulates cartilage matrix degradation in human osteoarthritis. J Mol Med . 2016;94(7):771-785.

25. Arner EC, Tortorella MD. Signal transduction through chondrocyte integrin receptors induces matrix metalloproteinase synthesis and synergizes with interleukin-1. Arthritis Rheum. 1995;38(9):1304-1314.

26. Fernandes JC, Martel-Pelletier J, Pelletier J-P. The role of cytokines in osteoarthritis pathophysiology. Biorheology. 2002;39(1-2):237-246.

27. Li H, Wang D, Yuan Y, Min J. New insights on the MMP13 regulatory network in the pathogenesis of early osteoarthritis. Arthritis Res Ther. 2017;19(1):248.

28. Ji B, Ma Y, Wang H, Fang X, Shi P. Activation of the P38/ CREB/MMP13 axis is associated with osteoarthritis. Drug Des Devel Ther. 2019;13:2195-2204.

29. Tachmazidou I, Hatzikotoulas K, Southam L, et al. Identification of new therapeutic targets for osteoarthritis through genome-wide analyses of UK Biobank data. Nat Genet. 2019;51(2):230-236.

30. Choi M-C, Jo J, Park J, Kang HK, Park Y. NF-кB Signaling Pathways in Osteoarthritic Cartilage Destruction. Cells. 2019;8(7). doi:10.3390/cells8070734

31. Haag J, Gebhard PM, Aigner T. SOX gene expression in human osteoarthritic cartilage. Pathobiology. 2008;75(3):195-199.

32. Chou C-H, Lee MTM, Song I-W, et al. Insights into osteoarthritis progression revealed by analyses of both knee tibiofemoral compartments. Osteoarthritis Cartilage. 2015;23(4):571-580.

33. arcOGEN Consortium, arcOGEN Collaborators, Zeggini E, et al. Identification of new susceptibility loci for osteoarthritis (arcOGEN): a genome-wide association study. Lancet. 2012;380(9844):815-823.

34. Hatakeyama Y, Tuan RS, Shum L. Distinct functions of BMP4 and GDF5 in the regulation of chondrogenesis. $J$ Cell Biochem. 2004;91(6):1204-1217. 
35. Yao X, Zhang J, Jing X, et al. Fibroblast growth factor 18 exerts anti-osteoarthritic effects through PI3K-AKT signaling and mitochondrial fusion and fission. Pharmacol Res. 2019;139:314-324.

36. Caron MMJ, Emans PJ, Surtel DAM, van der Kraan PM, van Rhijn LW, Welting TJM. BAPX-1/NKX-3.2 Acts as a Chondrocyte Hypertrophy Molecular Switch in Osteoarthritis. Arthritis \& Rheumatology. 2015;67(11):29442956.

37. Motomura H, Seki S, Shiozawa S, Aikawa Y, Nogami M, Kimura T. A selective c-Fos/AP-1 inhibitor prevents cartilage destruction and subsequent osteophyte formation. Biochem Biophys Res Commun. 2018;497(2):756-761.

38. Hwang HS, Lee MH, Choi MH, Kim HA. NOD2 signaling pathway is involved in fibronectin fragment-induced pro-catabolic factor expressions in human articular chondrocytes. BMB Rep. 2019;52(6):373-378.

39. Wang T, He C. Pro-inflammatory cytokines: The link between obesity and osteoarthritis. Cytokine Growth Factor Rev. 2018;44:38-50.

40. Kim HA, Cho M-L, Choi HY, et al. The catabolic pathway mediated by Toll-like receptors in human osteoarthritic chondrocytes. Arthritis Rheum. 2006;54(7):2152-2163.

41. Sun H-Y, Hu K-Z, Yin Z-S. Inhibition of the p38-MAPK signaling pathway suppresses the apoptosis and expression of proinflammatory cytokines in human osteoarthritis chondrocytes. Cytokine. 2017;90:135-143.

42. van den Bosch MHJ, van Lent PLEM, van der Kraan PM. Identifying effector molecules, cells, and cytokines of innate immunity in OA. Osteoarthritis Cartilage. 2020;28(5):532-543.

43. Wojdasiewicz P, Poniatowski ŁA, Szukiewicz D. The role of inflammatory and anti-inflammatory cytokines in the pathogenesis of osteoarthritis. Mediators Inflamm. 2014;2014:561459.

44. Pelletier JP, McCollum R, Cloutier JM, Martel-Pelletier J. Synthesis of metalloproteases and interleukin 6 (IL-6) in human osteoarthritic synovial membrane is an IL-1 mediated process. J Rheumatol Suppl. 1995;43:109-114.

45. Lu X, Gilbert L, He X, Rubin J, Nanes MS. Transcriptional Regulation of the Osterix (Osx, Sp7) Promoter by Tumor Necrosis Factor Identifies Disparate Effects of Mitogen-activated Protein Kinase and NFkB Pathways. J Biol Chem. 2006;281(10):6297-6306.

46. Murphy G, Knäuper V, Atkinson S, et al. Matrix metalloproteinases in arthritic disease. Arthritis Res. 2002;4 Suppl 3:S39-S49.

47. Mohit Kapoor, Johanne Martel-Pelletier, Daniel Lajeunesse, Jean-Pierre Pelletier, Hassan Fahmi. Role of proinflammatory cytokines in the pathophysiology of osteoarthritis. Nature Reviews Rheumatology. https:// www.nature.com/articles/nrrheum.2010.196. Published
November 30, 2010. Accessed April 13, 2020.

48. Karlsson C, Dehne T, Lindahl A, et al. Genome-wide expression profiling reveals new candidate genes associated with osteoarthritis. Osteoarthritis Cartilage. 2010;18(4):581-592.

49. Chou C-H, Lee C-H, Lu L-S, et al. Direct assessment of articular cartilage and underlying subchondral bone reveals a progressive gene expression change in human osteoarthritic knees. Osteoarthritis Cartilage. 2013;21(3):450-461.

50. Zeltz C, Gullberg D. The integrin-collagen connection--a glue for tissue repair? J Cell Sci. 2016;129(4):653-664.

51. Homandberg GA, Hui F, Wen C, et al. Fibronectin-fragment-induced cartilage chondrolysis is associated with release of catabolic cytokines. Biochem J. 1997;321 ( Pt 3):751-757.

52. Mathiessen A, Conaghan PG. Synovitis in osteoarthritis: current understanding with therapeutic implications. Arthritis Res Ther. 2017;19(1):18.

53. de Lange-Brokaar BJE, Ioan-Facsinay A, van Osch GJVM, et al. Synovial inflammation, immune cells and their cytokines in osteoarthritis: a review. Osteoarthritis Cartilage. 2012;20(12):1484-1499.

54. Roman-Blas JA, Jimenez SA. NF-kappaB as a potential therapeutic target in osteoarthritis and rheumatoid arthritis. Osteoarthritis Cartilage. 2006;14(9):839-848.

55. Li J, Cao F, Yin H-L, et al. Ferroptosis: past, present and future. Cell Death Dis. 2020;11(2):88.

56. Collins JA, Arbeeva L, Chubinskaya S, Loeser RF. Articular chondrocytes isolated from the knee and ankle joints of human tissue donors demonstrate similar redox-regulated MAP kinase and Akt signaling. Osteoarthritis Cartilage. 2019;27(4):703-711.

57. Li T, Chubinskaya S, Esposito A, et al. TGF- $\beta$ type 2 receptor-mediated modulation of the IL-36 family can be therapeutically targeted in osteoarthritis. Sci Transl Med. 2019;11(491). doi:10.1126/scitranslmed.aan2585

58. Sandoval J, Pereda J, Pérez S, et al. Epigenetic Regulation of Early- and Late-Response Genes in Acute Pancreatitis. J Immunol. 2016;197(10):4137-4150.

59. Nilsson R, Bajic VB, Suzuki H, et al. Transcriptional network dynamics in macrophage activation. Genomics. 2006;88(2):133-142.

60. Tsukahara Y, Lian Z, Zhang X, et al. Gene expression in human neutrophils during activation and priming by bacterial lipopolysaccharide. J Cell Biochem. 2003;89(4):848-861.

61. Smale ST. Selective transcription in response to an inflammatory stimulus. Cell. 2010;140(6):833-844.

62. Naik E, Dixit VM. Mitochondrial reactive oxygen species drive proinflammatory cytokine production. J Exp Med. 
bioRxiv preprint doi: https://doi.org/10.1101/2020.06.18.155390; this version posted June 18, 2020. The copyright holder for this preprint (which was not certified by peer review) is the author/funder, who has granted bioRxiv a license to display the preprint in perpetuity. It is made available under aCC-BY-NC-ND 4.0 International license.

\section{1;208(3):417-420.}

63. Serrat N, Sebastian C, Pereira-Lopes S, Valverde-Estrella L, Lloberas J, Celada A. The response of secondary genes to lipopolysaccharides in macrophages depends on histone deacetylase and phosphorylation of C/EBP $3 . J \mathrm{Im}$ munol. 2014;192(1):418-426.

64. Tullai JW, Schaffer ME, Mullenbrock S, Sholder G, Kasif S, Cooper GM. Immediate-early and delayed primary response genes are distinct in function and genomic architecture. J Biol Chem. 2007;282(33):23981-23995.

65. O’Donnell VB, Aldrovandi M, Murphy RC, Krönke G. Enzymatically oxidized phospholipids assume center stage as essential regulators of innate immunity and cell death. Sci Signal. 2019;12(574). doi:10.1126/scisignal.aau2293

66. Shah R, Raska K Jr, Tiku ML. The presence of molecular markers of in vivo lipid peroxidation in osteoarthritic cartilage: a pathogenic role in osteoarthritis. Arthritis Rheum. 2005;52(9):2799-2807.

67. Carlo MD Jr, Loeser RF. Increased oxidative stress with aging reduces chondrocyte survival: correlation with intracellular glutathione levels. Arthritis Rheum. 2003;48(12):3419-3430.

68. Blanco FJ, Rego I, Ruiz-Romero C. The role of mitochondria in osteoarthritis. Nat Rev Rheumatol. 2011;7(3):161169.

69. Martel-Pelletier J, Lajeunesse D, Reboul P, Pelletier J-P. Therapeutic role of dual inhibitors of 5-LOX and COX, selective and non-selective non-steroidal anti-inflammatory drugs. Ann Rheum Dis. 2003;62(6):501-509.

70. Carroll GJ, Breidahl WH, Bulsara MK, Olynyk JK. Hereditary hemochromatosis is characterized by a clinically definable arthropathy that correlates with iron load. Arthritis Rheum. 2011;63(1):286-294.

71. Hochberg MC, Guermazi A, Guehring H, et al. Effect of Intra-Articular Sprifermin vs Placebo on Femorotibial Joint Cartilage Thickness in Patients With Osteoarthritis: The FORWARD Randomized Clinical Trial. JAMA. 2019;322(14):1360-1370.

72. Southam L, Rodriguez-Lopez J, Wilkins JM, et al. An SNP in the 5'-UTR of GDF5 is associated with osteoarthritis susceptibility in Europeans and with in vivo differences in allelic expression in articular cartilage. Hum Mol Genet. 2007;16(18):2226-2232.

73. Wang RN, Green J, Wang Z, et al. Bone Morphogenetic Protein (BMP) signaling in development and human diseases. Genes Dis. 2014;1(1):87-105.

74. Ishida W, Hamamoto T, Kusanagi $\mathrm{K}$, et al. Smad6 is a Smad1/5-induced smad inhibitor. Characterization of bone morphogenetic protein-responsive element in the mouse Smad6 promoter. J Biol Chem. 2000;275(9):60756079 .
75. Scharstuhl A, Vitters EL, van der Kraan PM, van den Berg WB. Reduction of osteophyte formation and synovial thickening by adenoviral overexpression of transforming growth factor beta/bone morphogenetic protein inhibitors during experimental osteoarthritis. Arthritis Rheum. 2003;48(12):3442-3451.

76. D’Costa S, Rich MJ, Diekman BO. Engineered Cartilage from Human Chondrocytes with Homozygous Knockout of Cell Cycle Inhibitor p21. Tissue Eng Part A. 2020;26(78):441-449. 
bioRxiv preprint doi: https://doi.org/10.1101/2020.06.18.155390; this version posted June 18, 2020. The copyright holder for this preprint (which was not certified by peer review) is the author/funder, who has granted bioRxiv a license to display the preprint in perpetuity. It is made available under aCC-BY-NC-ND 4.0 International license.

\section{Supplemental Figures}

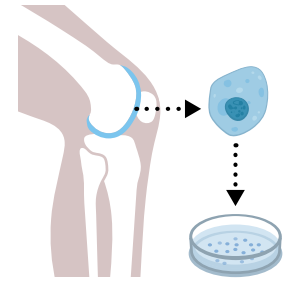

Isolation

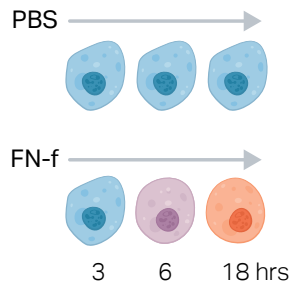

Treatment

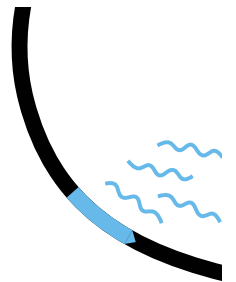

RNA-seq
Figure S1. Experimental design of fibronectin fragment (FN-f) treatment. Primary articular chondrocytes were isolated from normal human femoral cartilage obtained from three tissue donors aged 50-61 years, each without osteoarthritis. Six samples from each donor were cultured and treated with either $1 \mu \mathrm{M}$ fibronectin fragments (FN-f) or PBS. After 3, 6, or 18 hours, RNA was extracted and used to create RNA-seq libraries. This design allowed for the comparison of gene expression in FN-f-treated samples compared to time-matched untreated controls, accounting for changes occurring as a result of isolation, time in culture, and donor genotype.

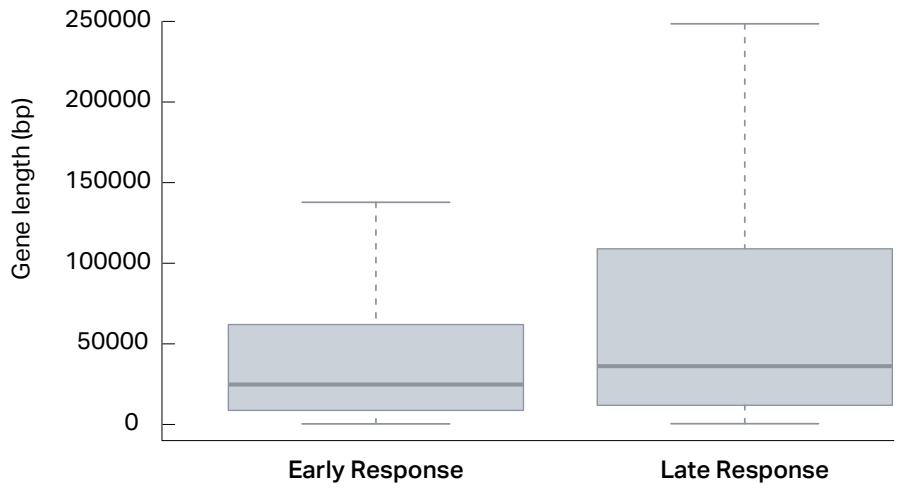

Figure S2. Gene length distributions among genes up- and downregulated by FN-f. Boxplots depicting the lengths of early-response genes (both up and down, $n=696$ ) and late-response genes (both up and down, $n=528$ ) reveal that late-response genes are statistically significantly longer (Mann-Whitney U test, p-value $<0.0001$ ). Outliers are excluded from this plot.

\section{De Novo Motifs in Differential Gene Promoters}

Motif $\begin{aligned} & \text { P-value } \\ & \text { Cluster }\end{aligned}$

Figure S3. Unannotated de novo motifs identified in differential gene promoters. When the promoters of differential genes among each cluster were analyzed for enriched transcription factor motifs, several had no clear match to known motifs (motif match score $<0.6$ ). These unannotated de novo motifs represent potential binding sites of transcription factors that have yet to be characterized. For each motif, the motif logo, p-value of enrichment, and cluster in which it was enriched are listed.
Table S1. Differential genes in response to FN-f treatment

This table lists all differential genes $(n=1,224)$ that change in response to FN-f treatment. For each gene, the response class is listed (as determined by k-means clustering of all differential genes), as well as the p-value (Wald test), log2 fold-change, and z-score normalized counts at each time point, and the raw counts from each sample.

Table S2. Osteoarthritis genes in response to FN-f treatment

This table includes all OA-responsive genes from the RAAK study with a p-value less than 0.01 and an absolute fold-change greater than $1.5(n=87)$, and details how they change in response to FN-f treatment. For each gene, the response class is listed (as determined by k-means clustering of all differential genes), as well as the p-value (Wald test), log2 foldchange, and z-score normalized counts at each time point, the raw counts from each sample, and the original $\mathrm{p}$-value and foldchange from the RAAK study [19]. 